\title{
Expected Performance of the ATLAS Inner Detector
}

\author{
Sofia Chouridou ${ }^{1}$ \\ University of California, Santa Cruz \\ Santa Cruz UC, SCIPP, 1156 High Street, Santa Cruz CA 95064, USA \\ E-mail: Sofia.Chouridouacern.ch
}

On behalf of the ATLAS Collaboration

\begin{abstract}
The ATLAS experiment at the CERN Large Hadron Collider (LHC) has started taking data in September 2008 with the inauguration of the LHC. Since then, the detector has been commissioned and calibrated in the ATLAS cavern. The ATLAS Inner Detector (ID) placed within a $2 \mathrm{~T}$ solenoid magnetic field, is a tracking system for charged particles based on three technologies: silicon pixels, silicon micro-strips and straw drift tubes. The LHC physics discovery potential sets stringent requirements on the performance of the ID, specially in terms of pattern recognition, tracking, momentum and vertex resolution and low radiation length to minimize the material effects. After a brief description of the detector design, the expected performance, based on Monte Carlo data, for identifying and reconstructing single particles with transverse momentum $\mathrm{p}_{\mathrm{T}}>0.5 \mathrm{GeV}$ is described as well as the detector's vertexing and btagging capabilities. Finally, cosmic ray results that verify so far that the detector is ready to fulfill the expected performance specifications are outlined.
\end{abstract}

12th International Conference on B-Physics at Hadron Machines - BEAUTY 2009

Heidelberg, Germany

September 07-11, 2009

${ }^{1}$ Speaker 


\section{Introduction}

The ATLAS detector [1] is a multi-purpose detector at the Large Hadron Collider (LHC) at CERN. Physics at the harsh environment of the LHC (design luminosity of $10^{34} \mathrm{~cm}^{-2} \mathrm{~s}^{-1}$ ), imposes stringent requirements on the measurement of the particle momentum and vertex resolution. To achieve these requirements high precision measurements will be made in the Inner Detector (ID), shown in Fig. 1.

The ATLAS ID surrounds the LHC beam-pipe which is inside a radius of $36 \mathrm{~mm}$ and it is immersed in a $2 \mathrm{~T}$ magnetic field provided by the central solenoid. It consists of three subsystems: silicon pixels, silicon micro-strip layers (Semi Conductor Tracker or SCT) and straw tube elements (Transition Radiation Tracker or TRT).

The pixels and SCT cover the region of $|\eta|<2.5^{1}$. In the barrel region they are arranged on concentric cylinders around the beam axis while in the end-cap regions, they are located on disks perpendicular to the beam axis.

The minimum pixel size on a sensor is $50 \times 400 \mu \mathrm{m}^{2}$. The intrinsic accuracies of the position measurement in the barrel are $10 \mu \mathrm{m}$ in the transverse $(\mathrm{R}-\varphi)$ plane and $115 \mu \mathrm{m}$ along the beamline $(\mathrm{z})$, while in the disks are $10 \mu \mathrm{m}(\mathrm{R}-\varphi)$ and $115 \mu \mathrm{m}$ along the radial distance from the beamline $(\mathrm{R})$. The total number of readout channels of the pixel detector is 80.4 million.

For the SCT, small-angle (40 mrad) stereo strips with a strip pitch of $80 \mu \mathrm{m}$ are used to measure both coordinates. The intrinsic accuracies per SCT module in the barrel are typically $16 \mu \mathrm{m}(\mathrm{R}-\varphi)$ and $580 \mu \mathrm{m}(\mathrm{z})$ and in the disks are $16 \mu \mathrm{m}(\mathrm{R}-\varphi)$ and $580 \mu \mathrm{m}(\mathrm{R})$. The SCT has approximately 6.3 million readout channels.

The TRT consists of $4 \mathrm{~mm}$ diameter straw tubes filled with a xenon-based gas mixture at overpressure of 5-10 mbar. It covers the region of up to $|\eta|=2.0$ and provides only $\mathrm{R}-\varphi$ information with an intrinsic accuracy of $130 \mu \mathrm{m}$ per straw. The total number of TRT readout channels is approximately 350 thousand. The combination of precision trackers at small radii with the TRT at a larger radius gives very robust pattern recognition and very precise reconstruction of tracks with transverse momentum $\mathrm{p}_{\mathrm{T}}>$ $0.5 \mathrm{GeV}$ and $|\eta|<2.5$.

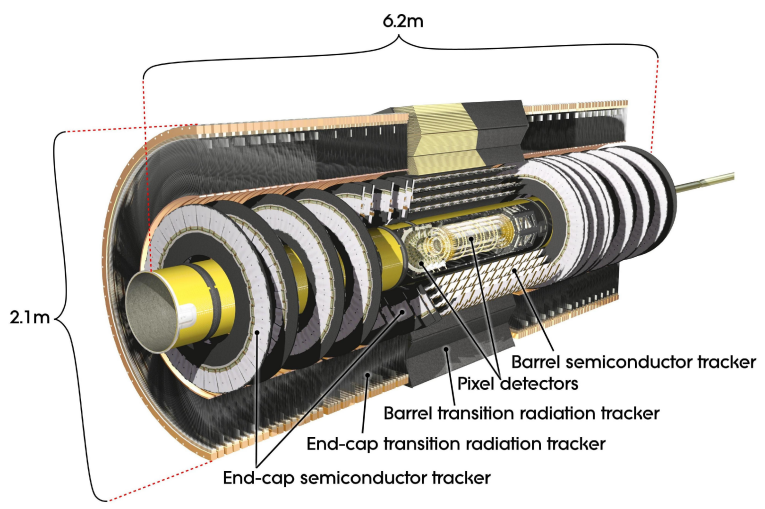

Figure 1: The ATLAS Inner Detector.

\footnotetext{
${ }^{1}$ The pseudorapidity $\eta$ is defined as $\eta=-\ln \tan \theta / 2$ where $\theta$ is the angle with respect to the beamline.
} 


\section{Tracking Performance}

The ATLAS tracking software [2] is flexible and modular in design. It uses a common event data model which allows for standardised interfaces to all reconstruction tools, such as track extrapolation, track fitting including material corrections and vertex finding and fitting.

First the raw data are converted into clusters (pixel/SCT) and drift circles (TRT). Track finding is performed in two directions: inside-out, where selected tracks in the pixel and SCT are extended into the TRT and outside-in (back-tracking), where unused TRT track segments are extended into the silicon detectors to improve the tracking efficiency for secondary tracks from conversions or decays of longlived particles. Finally, the primary, as well as the secondary vertices are reconstructed.

\subsection{Track parameter resolutions}

The resolution of a track parameter X (inverse transverse momentum, azimuthal angle, polar angle, transverse impact parameter and longitudinal impact parameter) can be expressed as a function of the $\mathrm{p}_{\mathrm{T}}$ [3] as:

$$
\sigma_{\mathrm{X}}\left(\mathrm{p}_{\mathrm{T}}\right)=\sigma_{\mathrm{X}}(\infty)\left(1 \oplus \mathrm{p}_{\mathrm{X}} / \mathrm{p}_{\mathrm{T}}\right)
$$

where $\sigma_{\mathrm{X}}(\infty)$ is the asymptotic resolution expected at infinite momentum, $\mathrm{p}_{\mathrm{X}}$ is a constant representing the value of $\mathrm{p}_{\mathrm{T}}$ for which the intrinsic and multiple scattering terms in the equation are equal for the parameter $\mathrm{X}$ under consideration and $\oplus$ denotes addition in quadrature.

Figures 2 and 3 show the momentum resolution and the transverse impact parameter resolution for isolated muons of different transverse momenta.

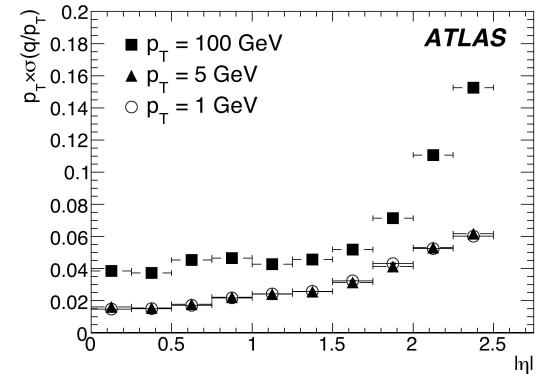

Figure 2: Relative transverse momentum resolution as a function of $I \eta \mid$ for muons with $\mathrm{p}_{\mathrm{T}}=1,5$ and $100 \mathrm{GeV}$.

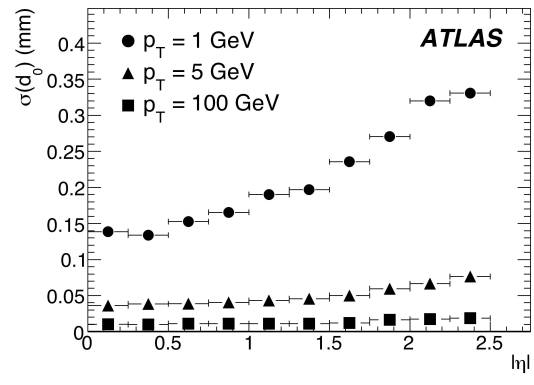

Figure 3: Transverse impact parameter, $\mathrm{d}_{0}$, resolution as a function of $I \eta \mid$ for muons with $\mathrm{p}_{\mathrm{T}}=1,5$ and $100 \mathrm{GeV}$.

\subsection{Track reconstruction efficiency}

The tracking efficiency is defined as the fraction of truth particles which are matched to reconstructed tracks passing the following quality cuts: only prompt particles with $\mathrm{p}_{\mathrm{T}}>1 \mathrm{GeV}$ and $|\eta|<$ 2.5 are considered with tracks that have at least seven precision hits (pixels and SCT) and their transverse and longitudinal impact parameters at the perigee fulfil respectively $\left|d_{0}\right|<2 \mathrm{~mm}$ and $\left|\mathrm{z}_{0}-\mathrm{z}_{\nu}\right| \times \sin \theta<10$ $\mathrm{mm}$, where $z_{v}$ is the position of the primary vertex along the beam and $\theta$ is the polar angle of the track. According to stricter selection cuts, called b-tagging quality cuts, the tracks should have at least two hits in the pixel layers, one of which should be in the innermost pixel layer and their impact parameters should be $\left|d_{0}\right|<1 \mathrm{~mm}$ and $\left|z_{0}-z_{\nu}\right| \times \sin \theta<1.5 \mathrm{~mm}$. The efficiency for reconstructing isolated single 
particles (muons, pions and electrons) are shown in Figure 4. The curves for pions and electrons reflect the shape of the amount of ID material, as in addition to multiple scattering, pions are affected by hadronic interactions in the material and electrons emit bremsstrahlung radiation. Figure 5 shows the tracking efficiency for prompt pions and the fake rates for tracks in jets in events containing top-quark pairs as a function of $|\eta|$. The fake rate is defined as the fraction of reconstructed tracks passing the quality cuts which are not matched to a particle.

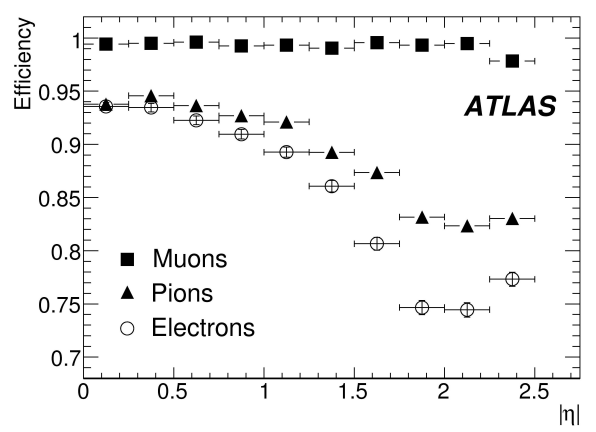

Figure 4: Track reconstruction efficiencies as a function of $|\eta|$ for muons, pions and electrons with $\mathrm{p}_{\mathrm{T}}=5 \mathrm{GeV}$.

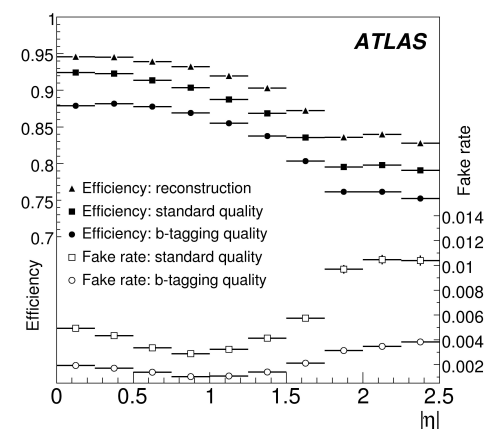

Figure 5: Track reconstruction efficiencies and fake rates as a function of $|\eta|$ for charged pions in jets in ttbar events for different quality cuts.

\section{Vertexing Performance}

\subsection{Primary vertices}

The precise reconstruction of primary vertices is crucial for many physics studies like searches for new particles, tagging of $b$ - and $\tau$-jets, reconstruction of exclusive b-decays and similar applications. The resolutions of the primary vertex reconstruction along $\mathrm{x}$, in the transverse plane and along $\mathrm{z}$, parallel to the beam axis for $\mathrm{H} \rightarrow \gamma \gamma$ decays with $\mathrm{m}_{\mathrm{H}}=120 \mathrm{GeV}$ and for events containing top-quark pairs are shown in Figures 6 and 7.

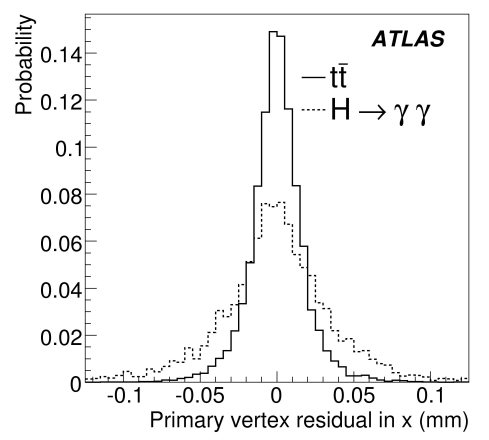

Figure 6: Primary vertex resolution along $\mathrm{x}$ for ttbar $(\mathrm{RMS}=18 \mu \mathrm{m})$ and $\mathrm{H} \rightarrow \gamma \gamma(\mathrm{RMS}=36 \mu \mathrm{m})$ events with $\mathrm{m}_{\mathrm{H}}=120 \mathrm{GeV}$.

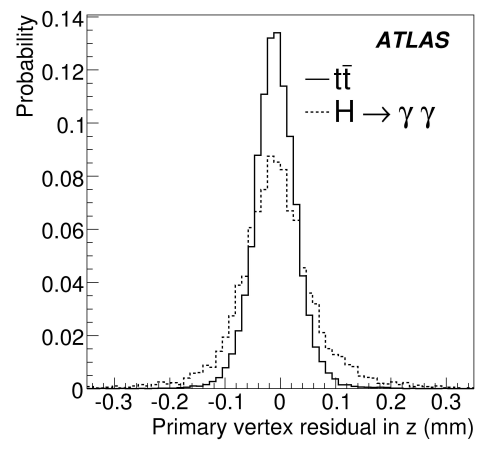

Figure 7: Primary vertex resolution along $\mathrm{z}$ for ttbar $(\mathrm{RMS}=41 \mu \mathrm{m})$ and $\mathrm{H} \rightarrow \gamma \gamma(\mathrm{RMS}=72 \mu \mathrm{m})$ events with $\mathrm{m}_{\mathrm{H}}=120 \mathrm{GeV}$. 


\subsection{Secondary Vertices}

The resolution for the reconstruction of the radial position of secondary vertices for the decays of $\mathrm{J} / \psi \rightarrow \mu \mu$ in events containing B-hadron decays is presented in Figures 8 and 9. In these decays the average transverse momentum of the $\mathrm{J} / \psi$ is $15 \mathrm{GeV}$.

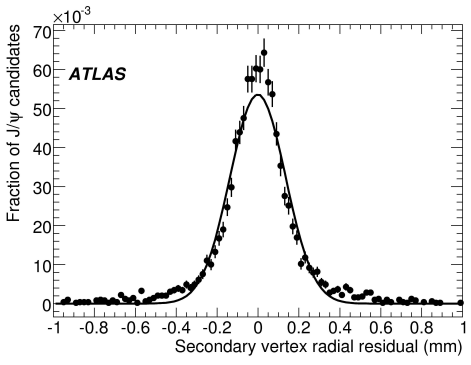

Figure 8: Resolution (width of the distribution) for the reconstruction of the radial position of the secondary vertex for $\mathrm{J} / \psi \rightarrow \mu \mu$ decays for tracks with $|\eta|$ around 0 .

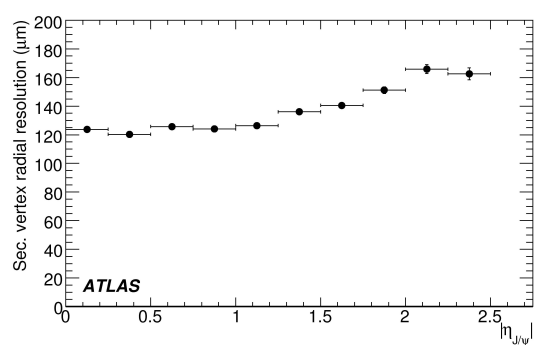

Figure 9: Resolution for the reconstruction of the radial position of the secondary vertex for $\mathrm{J} / \psi \rightarrow \mu \mu$ decays as a function of the pseudorapidity of the $\mathrm{J} / \psi$.

Figure 10 shows the resolution for the reconstructed radial position of the secondary vertex for $\mathrm{K}_{\mathrm{S}} \rightarrow \Pi^{+} \Pi^{-}$decays in events containing B-hadron decays as a function of the $\mathrm{K}_{\mathrm{S}}$ decay radius. The resolution is better for decays that take place just in front of the detector layers. The effect of crossing the three successive pixel layers is clearly visible as well as the degraded resolution for decays beyond the last pixel layer. The barrel pixel layers are at 51, 89 and $123 \mathrm{~mm}$, while the first two SCT layers are at 299 and $371 \mathrm{~mm}$. Therefore, the large increase at $\sim 115 \mathrm{~mm}$ is due to the $\sim 200 \mathrm{~mm}$ gap between the pixels and the SCT.

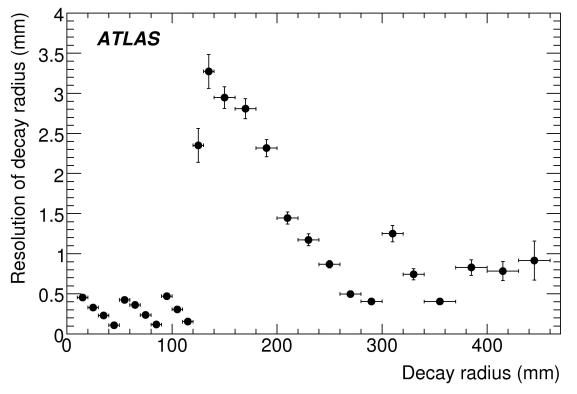

Figure 10: Radial position resolution of the secondary vertex $K_{S} \rightarrow \Pi^{+} \Pi^{-}$as a function of the $K_{S}$ decay radius.

Around $10-50 \%$ (depending on the pseudorapidity) of the photons produced in the collision, convert into an $\mathrm{e}^{+} \mathrm{e}^{-}$pair before leaving the SCT. The efficiencies to reconstruct a) single tracks from photon conversions, b) the pair of tracks from the conversion and c) the photon conversion vertex as a function of the conversion radius are presented in Figure 11. Only tracks originated from a radial distance from the beam axis of up to $800 \mathrm{~mm}$ can be efficiently reconstructed. Due to the more limited TRT resolution and the potentially missing silicon information at radial distances larger than $400 \mathrm{~mm}$, the efficiency for track pairs is reduced. The efficiency can be increased at large radii by identifying and flagging single electron tracks as photon conversions under certain conditions: a) the track is created 
inside the ID volume, b) there is no hit in the innermost pixel layer and c) there is transition radiation (Figure 12).

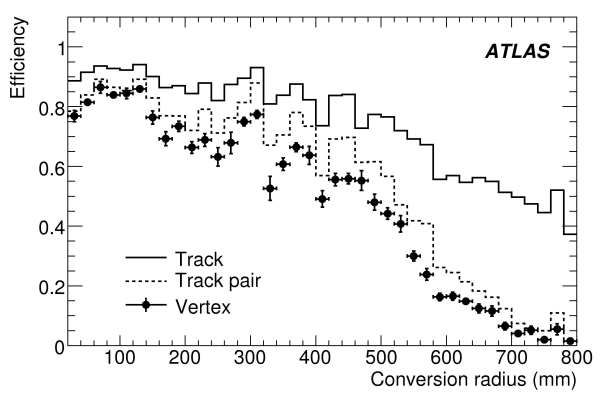

Figure 11: Efficiency to reconstruct conversions of photons with $\mathrm{p}_{\mathrm{T}}=20 \mathrm{GeV}$ and $|\eta|<2.1$, as a function of the conversion radius.

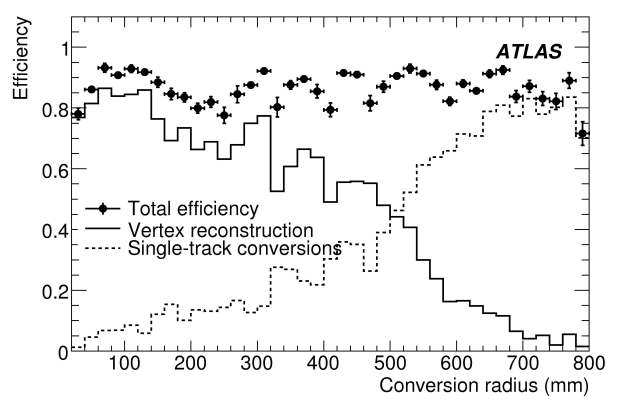

Figure 12: The total efficiency to identify conversions of photons is a combination of the vertex reconstruction efficiency (as shown in Figure 11) and the efficiency to identify singletrack conversions.

The identification of jets stemming from the hadronization of b quarks (b-tagging) is an important asset for many physics analysis (top, Higgs, SUSY, etc). This identification is based on several special properties of the B-hadrons like their relatively high $(>5 \mathrm{GeV})$ mass, long lifetime (resulting in a significant flight path length), large transverse momentum of their decay products with respect to the jet axis and large opening angle between these products. There are numerous algorithms (and their combinations) used in ATLAS for b-tagging. The b-jet efficiency is defined as the fraction of taggable jets labelled as b-jets which are actually tagged as b-jets by the tagging algorithm. The jet rejection is defined as the inverse of the mis-tagging rate (the fraction of taggable jets not labelled as $b$ which are actually tagged as b-jets). Finally, a light jet is defined as purified, when a b-quark, a c-quark or a $\tau$ lepton is not found within a cone of radius $\Delta \mathrm{R}=0.8$ around its direction ${ }^{2}$. Figure 13 shows the light and cjets rejection as a function of the the b-jet efficiency for events containing top-quark pairs. A light jet rejection higher than 100 can be achieved for a b-jet efficiency of $60 \%$. Note that the curves for c-jets without and with purification lie on top of each other.

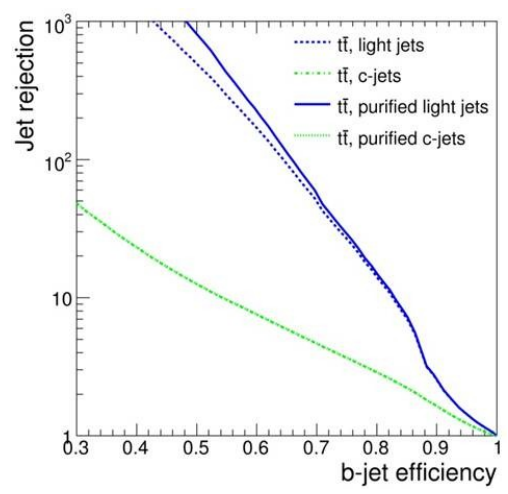

Figure 13: Rejection of light jets and c-jets with and without purification as a function of the b-tagging efficiency for events containing top-quark pairs.

\footnotetext{
${ }^{2}$ The distance $\Delta \mathrm{R}$ is defined as $\Delta \mathrm{R}=\sqrt{ } \Delta \eta^{2}+\Delta \varphi^{2}$, where $\Delta \eta$ is the difference between the pseudorapidities of the two relevant objects and $\Delta \varphi$ is the difference between their azimuthal angles.
} 


\section{Commissioning of the ID with Cosmic Rays}

Since the first LHC start-up in September 2008, the ATLAS detector has been continuously commissioned and calibrated in the cavern using cosmic rays. Figure 14 shows the unbiased residuals in the transverse plane $(\mathrm{R}-\varphi)$ for the pixel barrel [4]. The alignment of the detector is based on the minimization of the residuals in an iterative procedure. Upon convergence, the alignment constants (6 per silicon module) are stored [5]. The relative momentum resolution of the cosmic tracks as a function of the muon $\mathrm{p}_{\mathrm{T}}$ is shown in Figure 15. For this measurement, each reconstructed track was split in two halves giving two collision-like tracks. The differences to the Monte Carlo curve indicate the remaining misalignment.

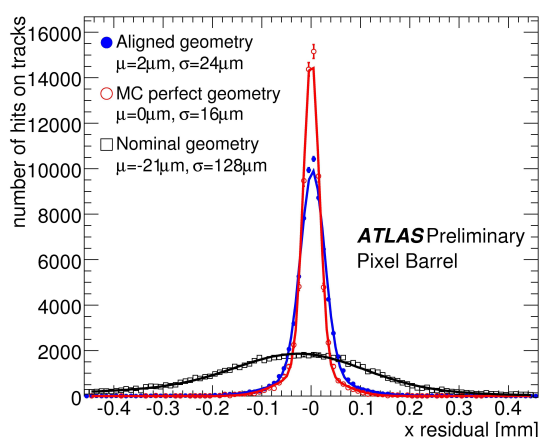

Figure 14: Pixel barrel residuals in the $\mathrm{R}-\varphi$ plane for three geometries: Monte Carlo perfect geometry, the nominal and the aligned.

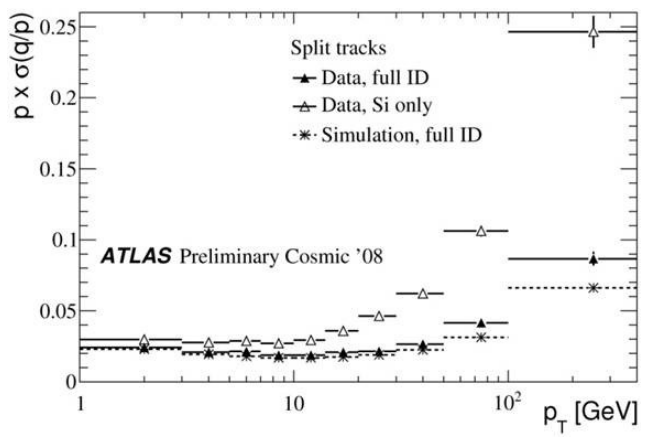

Figure 15: Relative momentum resolution as a function of the muon $\mathrm{p}_{\mathrm{T}}$ using only the silicon detector data (open triangles) and using the full ID data (filled triangles). The asterisks indicate the Monte Carlo simulation.

\section{Conclusion}

Physics at the LHC imposes stringent requirements on the ATLAS ID performance. Extensive simulations have been studying all the relevant parameters and issues. The cosmic ray results obtained during the detector commissioning in the cavern verify that the detector is ready to fulfill the expected performance specifications.

\section{References}

[1] The ATLAS Collaboration, G. Aad et al., The ATLAS Experiment at the CERN Large Hadron Collider, JINST 3 (2008) S08003.

[2] T. Cornelissen et al., Concepts, Design and Implementation of the ATLAS New Tracking, ATLAS Note ATLSOFT-PUB-2007-007, 2007.

[3] The ATLAS Collaboration, G. Aad et al., Expected Performance of the ATLAS experiment: detector, trigger and physics, CERN-OPEN-2008-020, 2008.

[4] The ATLAS Collaboration, A. Andreazza et al., Commissioning and performance of the ATLAS Inner Detector with the first beam and cosmic data, ATLAS Note ATL-INDET-PROC-2009-009, 2009.

[5] P. Bruckman de Renstrom, Alignment strategy for the Inner Detector of ATLAS, CERN-2007-004 pp. 147-158, 2007. 